\title{
Horizontal-Slot Plasmonic-Organic Hybrid (POH) Modulator
}

\author{
Sandeep Ummethala ${ }^{1,2, *}$, Venkata Anirudh Pammi', Ahsan H. M. Uddin ${ }^{1}$, Lothar Hahn², \\ Wolfgang Freude ${ }^{1}$, Christian Koos ${ }^{1,2, \dagger}$ \\ ${ }^{1}$ Institute of Photonics and Quantum Electronics (IPQ), Karlsruhe Institute of Technology (KIT), Karlsruhe, 76131 Germany \\ ${ }^{2}$ Institute of Microstructure Technology (IMT), Karlsruhe Institute of Technology (KIT), Eggenstein-Leopoldshafen, 76344, Germany \\ sandeep.ummethala@kit.edu, ${ }^{\dagger}$ christian.koos@kit.edu
}

Abstract: We demonstrate horizontal-slot plasmonic-organic hybrid (HS-POH) modulators and show that the $\pi$-voltage-loss product $a U_{\pi} L$ is by $25 \%$ better than for conventional vertical-slot devices. The slot is realized by a sacrificial layer, thereby relaxing extreme lithography-resolution

\section{Introduction} requirements. (C) 2020 The Author(s)

Plasmonic-organic hybrid $(\mathrm{POH})$ electro-optic modulators combine plasmonic slot waveguides with highly efficient organic electro-optic (EO) materials [1] to achieve high modulation efficiencies. The devices offer ultra-small footprint and lend themselves to co-integration with low-loss silicon photonic circuits [2]. As a prominent feature, POH modulators provide unprecedented EO bandwidths of hundreds of $\mathrm{GHz}$ [3] [4], thereby opening a route towards seamless conversion of data signals from $\mathrm{THz}$ carriers to optical carriers [3]. However, the practical application of POH modulators is still subject to various limitations. POH devices suffer from comparatively high propagation losses of the plasmonic slot waveguides, thereby limiting the interaction length and increasing the required modulation voltage. This effect is quantified by the $\pi$-voltage-loss product $a U_{\pi} L$, i. e., by the product of required $\pi$-voltage $U_{\pi}$ measured in $\mathrm{V}$ and the phase-shifter insertion loss $a \mathrm{~L}$ in $\mathrm{dB}$. Moreover, conventional POH modulators rely on precise fabrication of high aspect-ratio plasmonic slot waveguides using high-resolution lithography in combination with a sensitive lift-off process. Mitigation of these challenges would be an important step towards practical application of POH devices.

In this paper, we demonstrate a novel structure for $\mathrm{POH}$ electro-optic modulators, exploiting a horizontally oriented plasmonic slot. In contrast to lithographically defined conventional vertical-slot plasmonic waveguides, the basic structure of horizontal-slot POH (HS-POH) modulators is realized by spin-coating a thin sacrificial layer which allows to precisely adjust the slot dimensions, even without using high-resolution lithography. We demonstrate $\mathrm{HS}-\mathrm{POH}$ modulators with slot aspect ratios of more than 5, which allows decreasing the $\pi$-voltage-length product $U_{\pi} L$ by more than $40 \%$ in comparison to the vertical-slot structures. Based on electro-magnetic simulations, we show that the HSPOH configuration can reduce the $\pi$-voltage-loss product $a U_{\pi} L$ by more than $25 \%$. In addition, because the plasmonic section is side-coupled to silicon photonic strip waveguides, alignment tolerances can be relaxed without significantly affecting the coupling efficiency.

\section{Horizontal-slot Plasmonic-organic Hybrid (HS-POH) Mach-Zehnder Modulator}

The cross-section of a horizontal plasmonic waveguide, see Fig. 1(a), shows a sacrificial layer of patterned hydrogen silsesquioxane (HSQ) resist between two gold ( $\mathrm{Au}$ ) rails. When the HSQ layer is removed, the rails form a horizontal plasmonic slot waveguide. The use of HSQ allows for precise control of the spacing $d$ between the metal rails (width $r$ ) that form the horizontal plasmonic slot. In contrast to conventional vertical-slot POH (VS-POH) modulators, where the slot is defined by lithographic structuring and subsequent lift-off using a poly-methyl methacrylate (PMMA) mask, the HS-POH structure gives better control over the metal spacing $d$ and the flatness of the metal rails. Specifically, this configuration allows realizing plasmonic slot waveguides having significantly larger aspect ratios $r / d$ than those that are possible with conventional VS-POH devices where the aspect ratio is limited to about 3 . In the case of horizontalslot plasmonic waveguides, the maximum possible aspect ratio is limited only by the stability of the mechanical structure; an aspect ratio of 12 is feasible.

The plasmonic slot is filled with an EO polymer and guides a surface plasmon polariton (SPP). Light is coupled into the slot waveguide using a directional coupler section comprising a silicon ( $\mathrm{Si}$ ) strip waveguide in close proximity to the horizontal plasmonic slot, see Fig. 1(b). The Si waveguide carries a quasi-transverse-magnetic (TM) mode. By appropriately choosing the length of the directional coupler, a coupling efficiency of up to $90 \%$ is possible [5]. Such a side-coupling scheme for photonic-to-plasmonic mode conversion relaxes the alignment tolerances greatly. A falsecolored micrograph of an HS-POH Mach-Zehnder modulator (MZM) is shown in Fig. 1(c). Light is coupled in and out of the on-chip silicon photonic waveguides (green, width $500 \mathrm{~nm}$, height $220 \mathrm{~nm}$ ) via grating couplers (not shown), and a multi-mode interference (MMI) coupler is used to split the light to the two arms of the MZM. A geometrical path length difference of $80 \mu \mathrm{m}$ between the MZM arms allows for the selection of the operating point by tuning the wavelength. The RF signal is applied across the metal pads (dark yellow) with a ground-signal-ground (GSG) probe. Each arm of the MZM contains a phase-shifter section comprising two gold electrodes (bright yellow) with a rail width $r=400 \mathrm{~nm}$, which are separated by a distance $d=80 \mathrm{~nm}$, see Fig. 1(c) and inset. After fabrication of the HS-POH 
(a)

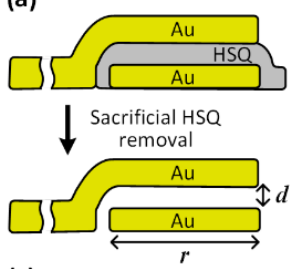

(c) (b)
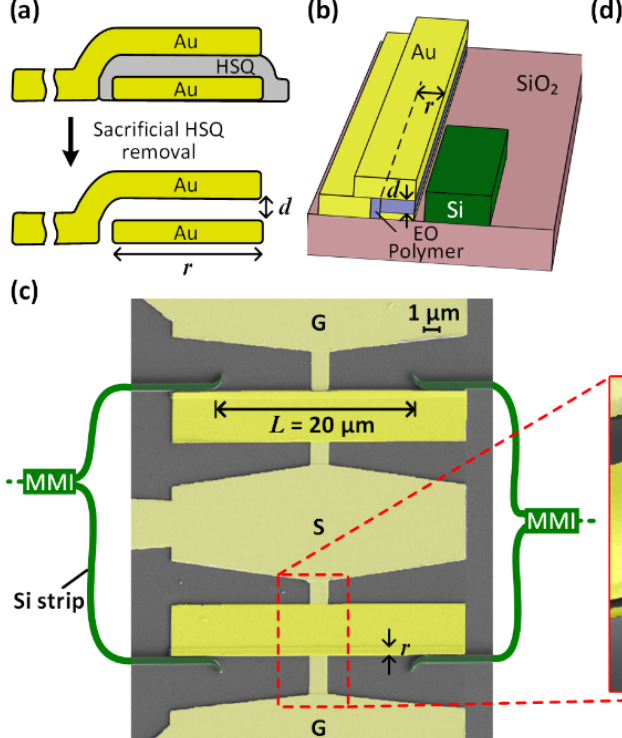

(d)
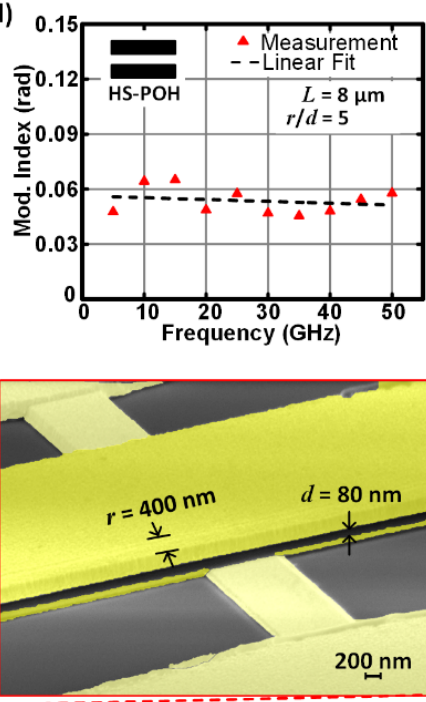

(e)

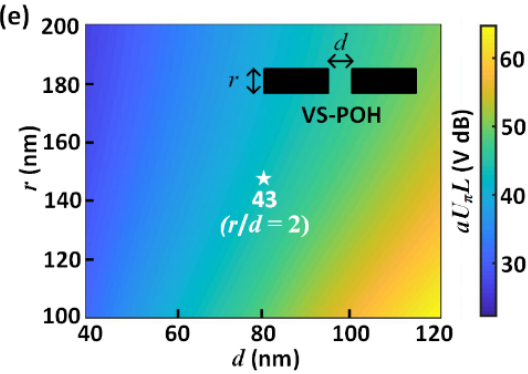

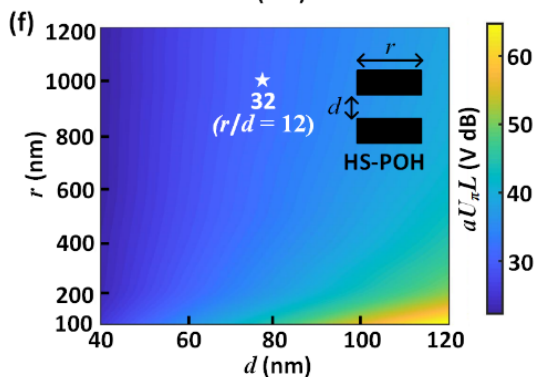

Figure 1: Horizontal-slot POH (HS-POH) Mach-Zehnder modulator (MZM). (a) HS plasmonic waveguide fabricated by using a sacrificial layer of HSQ resist. (b) Photonic-to-plasmonic converter with directional coupler comprising a Si strip and an HS plasmonic waveguide. (c) False-colored micrograph of HS-POH MZM showing GSG contact pads. The inset shows the gold rails with $r=400 \mathrm{~nm}$ separated by $d=80 \mathrm{~nm}$. (d) Frequency response of the modulation index (e, f) Color-coded $\pi$-voltage-loss product $a U_{\pi} L$ (in V dB) of VS-POH and VS$\mathrm{POH}$ modulators as a function of $d$ and $r$

MZM, the metal slots are filled with an organic EO polymer (SEO100) having a bulk EO coefficient $r_{33}$ of $166 \mathrm{pm} / \mathrm{V}$. For an $L=20 \mu \mathrm{m}$ long HS-POH MZM, we measure an insertion loss of $31 \mathrm{~dB}$ and an extinction ratio of more than $20 \mathrm{~dB}$. From dedicated cut-back measurements, we calculate a plasmonic loss of $1 \mathrm{~dB} / \mu \mathrm{m}$, a loss of $3.2 \mathrm{~dB}$ per mode converter, and a loss of $4.5 \mathrm{~dB}$ for the passive waveguides and MMIs. For a VS-POH modulator with $d=80 \mathrm{~nm}$ and an aspect ratio $r / d=1.9$, see inset Fig. 1(e), we measure $U_{\pi} L=12.6 \mathrm{~V}$, which reduces by $40 \%$ to $U_{\pi} L=7.6 \mathrm{~V}$ for an HS-POH modulator with the same $d$ but an aspect ratio of $r / d=5$, see inset Fig. 1(e). Figure 1(d) shows a flat frequency response of $L=8 \mu \mathrm{m}$ long HS-POH MZM up to $50 \mathrm{GHz}$ (limited by the presently available equipment), emphasizing the broadband characteristics of $\mathrm{POH}$ modulators. Using an electro-magnetic simulation tool, we calculate $a U_{\pi} L$ values for different geometrical variations of VS-POH and HS-POH modulators, see Figs. 1(e) and (f). For $d=80 \mathrm{~nm}$, the $\pi$-voltage-loss product can be reduced by $25 \%$ from $43 \mathrm{~V} \mathrm{~dB}$ to $32 \mathrm{~V}$ dB by increasing the aspect ratio $r / d$ from 2 for a vertical slot to 12 for a horizontal slot. The values of the $a U_{\pi} L$ products specified here are limited by the rather moderate EO coefficient $r_{33}=160 \mathrm{pm} / \mathrm{V}$ assumed in the calculations. By using more efficient EO materials [6] with $r_{33}$ values of $390 \mathrm{pm} / \mathrm{V}, a U_{\pi} L$ products down to $13 \mathrm{~V} \mathrm{~dB}$ can be achieved.

\section{Summary}

We realized a novel structure for POH electro-optic modulators, exploiting a horizontally oriented plasmonic slot. The concept stands out due to the significantly simplified fabrication process that requires neither high-resolution lithography nor a sensitive lift-off process. HS-POH modulators can be realized with large slot aspect ratios, thereby strongly reducing the $\pi$-voltage-loss product. We believe this robust design for POH modulators is a key step towards practical application of the devices.

This work was supported by the DFG project PACE (\# KO 4192/9-1) within the Priority Programme "Electronic-Photonic Integrated Systems for Ultrafast Signal Processing" (SPP 2111), by the European Research Council (ERC Consolidator Grant 'TeraSHAPE', \# 773248), by the Alfried Krupp von Bohlen und Halbach Foundation, and by the Karlsruhe Nano Micro Facility (KNMF). We thank Jingdong Luo and Alex K.-Y. Jen from Soluxra for providing the organic EO material.

\section{References}

[1] W. Heni et al., "Nonlinearities of organic electro-optic materials in nanoscale slots and implications for the optimum modulator design," vol. 25, no. 3, pp. 2627-2653, 2017.

[2] A. Melikyan et al., "Plasmonic-organic hybrid ( $\mathrm{POH})$ modulators for OOK and BPSK signaling at $40 \mathrm{Gbit} / \mathrm{s}, "$ Opt. Express, vol. 23, no. 8, pp. 9938-9946, 2015.

[3] S. Ummethala et al., "THz-to-optical conversion in wireless communications using an ultra-broadband plasmonic modulator," Nat. Photon., vol. 13, no. 8, pp. 519-524, 2019.
[4] M. Burla et al., "500 GHz plasmonic Mach-Zehnder modulator enabling sub-THz microwave photonics," APL Photonics, vol. 4, no. $5,2019$.

[5] A. Melikyan, M. Kohl, M. Sommer, C. Koos, W. Freude, and J. Leuthold, "Photonic-to-plasmonic mode converter," Opt. Lett., vol. 39 , no. 12, p. 3488, 2014.

[6] C. Kieninger et al., "Ultra-high electro-optic activity demonstrated in a silicon-organic hybrid ( $\mathrm{SOH})$ modulator," Optica, vol. 5, no. 6, pp. 739-748, 2018. 